\title{
STATUS OF THE WEST TRAVELLING WAVE ARRAY ANTENNA DESIGN AND RESULTS FROM THE HIGH POWER MOCK-UP
}

\author{
R. RAGONA, F. DURODIÉ, A. MESSIAEN, J. ONGENA, M. VAN SCHOOR \\ Laboratory for Plasma Physics, Royal Military Academy, Brussels, Belgium \\ Email: riccardo.ragona@mil.be
}

S. AGZAF, T. BATAL, J-M. BERNARD, X. COURTOIS, J-M. DELAPLANCHE, R. DUMONT, F. DURAND, F. FAISSE, M. FIRDAOUSS, L. GARGIULO, P. GARIBALDI, J. HILLAIRET, T. HOANG, G. LOMBARD, P. MOLLARD

CEA, IRFM, Saint Paul-lez-Durance, France

Email: julien.hillairet@cea.fr

Z. CHEN, Y. SONG, H. XU, Q. YANG, C. YU

ASIPP, Institute of Plasma Physics, Chinese Academy of Science, Hefei Anhui, China

Email: yangqx@ipp.ac.cn

\begin{abstract}
This paper presents the current status of the WEST TWA antenna, its mock-up and a possible extrapolation to DEMO. The updated WEST TWA design has a reduced antenna length and features feeding and mechanical support from a single vessel port. A mock-up of the WEST TWA antenna was designed in 2019, manufactured during 2020 and installed in the TITAN test facility at the beginning of 2021. The results of the mock-up at low and high power, its diagnostic system and the prospects are explained. Extensions towards a TWA antenna for WEST and a possible TWA system for the future DEMO tokamak reactor are briefly discussed.
\end{abstract}

\section{INTRODUCTION}

An Ion Cyclotron Range of Frequency (ICRF) system can provide power for several tasks [1], experimentally verified on present-day machines: heating and current drive, first wall conditioning [2], plasma start-up, removing of impurities from the core [3,4], controlling sawteeth [5] and current ramp down assist [6-8]. The system could be designed to have a high plug-to-power efficiency. Most of the components external to the machine are sturdy, however industrial steady-state capability requires still significant R\&D and qualification. Traditional ICRF antenna systems have evolved over decades, generally characterized by a high power density and often operated close to the voltage limits. However, low power density and low voltage would be very beneficial for the reliability of the system. Therefore, travelling wave type antennas have been proposed $[9,10]$ as a candidate ICRF system for a reactor. They could be integrated into the blanket, using only a limited number of feeders. Moreover, they could share the same cooling loop of the blanket thus reducing the integration complexity. Due to the larger number of toroidal straps, the $\mathrm{k}_{/ /}$spectrum is peaked and the dominant $\mathrm{k}_{/ /}$value can be optimized for coupling and bulk absorption while minimizing the interaction in the edge by depleting the low-k// part of the spectrum $[11,12]$ thus reducing the coaxial modes excitation and the dissipation in the RF sheaths. Depending on the configuration of the system, i.e. the number of arrays used, $50 \mathrm{MW}$ of RF power can be coupled to a plasma with an electron density profile given by the ITER-2010-low profile with a voltage on the antenna components of $\sim 15$ to $\sim 30 \mathrm{kV}[10,13,14]$. The larger the number of arrays, the lower the voltage thus reducing the risk for arcing phenomena and increasing the reliability of the system.

A proof of principle of the TWA concept for ICRH on a medium size tokamak is a needed step to consolidate the TWA conceptual design for tokamak reactors like DEMO or CFETR. The TWA concept was first presented in the early '90s [15] and tested only for fast wave current drive (FWCD) at high frequency [16]. More recently, the travelling wave 
antenna concept has been applied in the helicon current drive $[17,18]$ and the Lower Hybrid frequency range [19]. Notwithstanding this, a test for ICRH in a metal wall machine under steady-state operational conditions has never been done before. Those two conditions are fundamental for a reactor. For this reason, a test on the WEST machine is presently under consideration [20,21] with a high power mock-up as the first step [22]. To this end, a high RF power mock-up has been tested in the TITAN facility [23] to validate the RF design and the voltage stand-off.

This contribution presents the status of the WEST TWA antenna design, the preliminary results obtained from the high power mock-up, and the extrapolation towards a TWA antenna embedded in the breeding blanket of a tokamak reactor.

\section{WEST TWA}

The TWA antenna proposed in [20,21] made use of two vessel ports to allow using a long antenna with a geometry close to the proposed TWA DEMO antennas [13]. The antenna was $\sim 2.5 \mathrm{~m}$ long and was equipped with 8 straps. As the TWA antenna should ideally replace one of the currently installed WEST in-port antennas, it has been proposed to substitute the ICRH antenna located in sector Q4 allowing the use of the port in sector Q3 that was previously used by the electron cyclotron resonance heating (ECRH) system, which was decommissioned. However, in 2021 the WEST governing board decided to install a new ECRH system. The design of the TWA has thus been updated to comply with the new machine configuration. The new proposal will use only the port in sector Q4. To fit in the available space, the length of the antenna has been reduced. The number of straps has also been reduced to keep the same strap width as before together with the inter-strap distance and the lateral gaps. An overview of the updated design is presented in figure 1, which shows an equatorial cut of the WEST CAD model with the relative position of the installed ion cyclotron (IC) antennas, lower hybrid (LH) launchers and movable outboard limiter. It shows also the foreseen ECRH launcher and the proposed TWA (only the bottom row is shown in the figure).

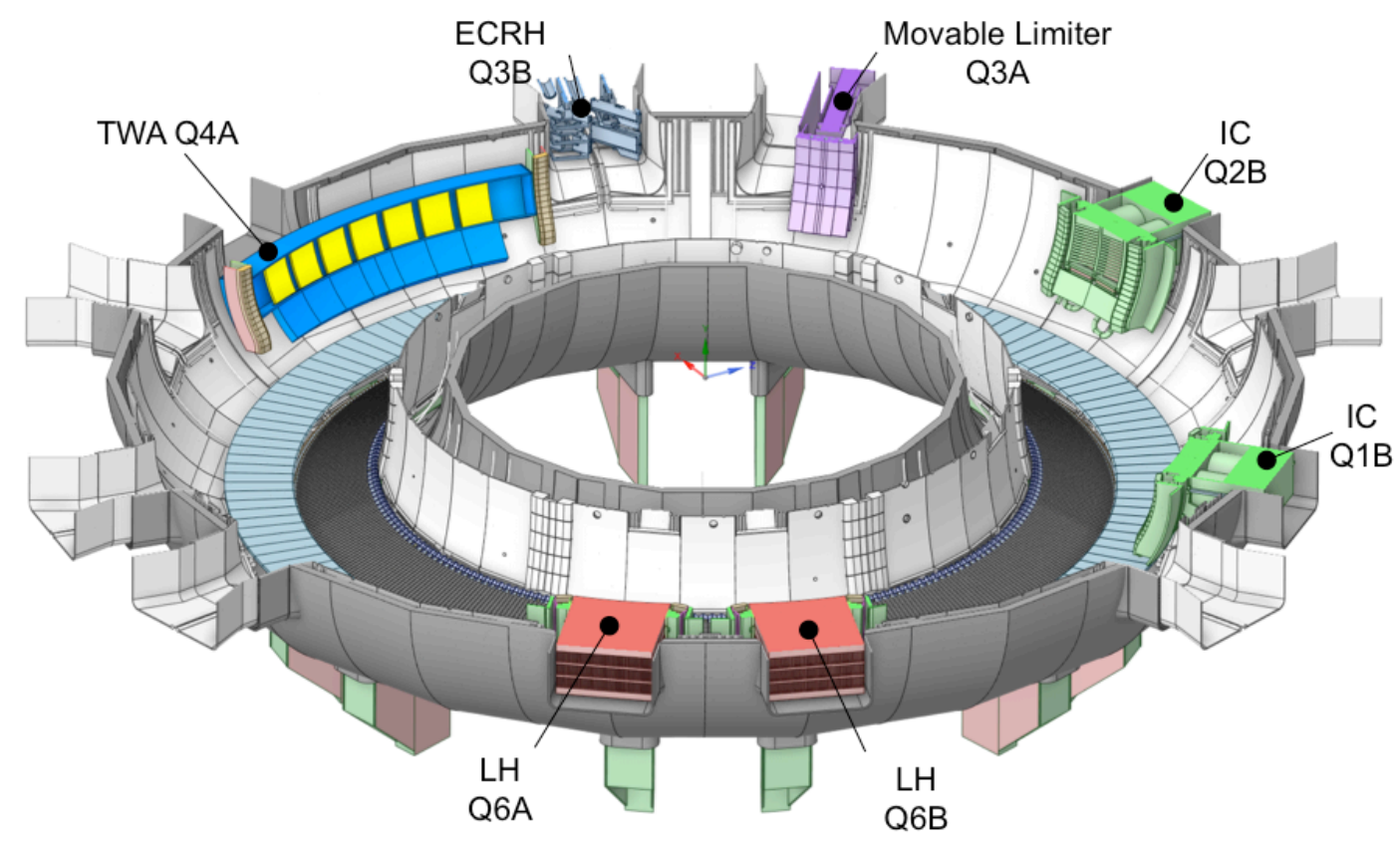


FIG. 1. Equatorial cut of the CAD model of WEST showing the installed Ion Cyclotron systems (IC Q1B, IC Q2B2), the Lower Hybrid launchers ( $L H$ Q6A, LH Q6B), and the outboard movable limiter. It also shows the foreseen Electron Cyclotron launcher and the location of the proposed TWA antenna (only the bottom row is shown).

It can be seen from the picture that the two lateral limiters on the TWA do not obstruct the two adjacent ports; one used for diagnostics and the other used by the ECRH system. In the case shown, the TWA uses a 7 straps configuration. A more detailed analysis is needed to assess the clearance between the ECRH beams and the lateral limiter of the TWA. The two TWA rows are displaced poloidally (see fig.8 in [22]). This allows increasing the decoupling between the two rows and at the same time allows the installation of the coaxial feeding lines. Moreover, additional diagnostic like RF field probes and reflectometers could be accommodated in the available space. The input and output feeding lines are routed through the single port used resulting in a four lines interface with the flange. Two lines are radially straight and two move toroidally to reach the other end of the two rows.

The presence of a large current loop across two machine segments in the previous design was identified as a potential risk. The large current loop was a consequence of the large toroidal extent of the antenna and the use of two ports, part of two different sections of the vessel, for its mechanical support. Large mechanical forces could be created in case of disruptions or vertical displacement events. This risk is now mitigated by the reduction of the antenna length and by the use of a single port. In addition, a system for radially displacing the antenna is now under consideration. In this new configuration where the antenna is fed from a single port, the external feeding circuit is not significantly changed. A resonant ring for each row could be located behind the port in sector Q4. The installation procedure of the TWA as studied in [22] is not negatively affected by the new design. The reduction of the number of straps by one unit has a small impact on the spectrum and results in a slightly larger recirculating fraction for the same coupled power. Nevertheless, further studies are required to assess the impact of the new configuration and to optimize its performance. They will be carried out during the next phase of the project. The option of equipping one of the rows with a Faraday shield is under consideration. This would allow a direct comparison between the two rows on the same plasma during the same experiment. The first results obtained with the mock-up, presented in this paper, provide confidence on the design for the WEST TWA and allow in addition the first extrapolation for a TWA in view of a future fusion reactor.

\section{TWA MOCKUP}

The antenna mock-up has been initially designed based on the specifications defined by the requirements for a WEST in-vessel component. Particular attention has been devoted to estimate the (i) plasma heat and particle loads, (ii) forces and momenta caused by vertical displacement events (VDE), (iii) RF losses, (iv) impact of the magnetic field ripple on the performance of the antenna and power deposition performances and profiles. The results have been used to dimension the relevant components of the WEST antenna, e.g. the strap thickness and the strap recess from the antenna aperture. However, the high power mock-up differs to the WEST TWA antenna. In particular, the mock-up consists of only 6 straps (to fit into the vacuum vessel), is flat instead of being conformal to the poloidal and toroidal curvature of the device, and is not water-cooled. Those differences have a negligible impact on the characteristics that are the purpose of the tests. Instead, they allowed simplifying the manufacturing process leading to a reduced cost for the procurement. The manufacturing of the mock-up has been carried out by ASIPP and has been delivered to CEA at the end of October 2020. 
Figure 2 shows the mock-up inside TITAN [23] after its installation, which took place at the end of March 2021. The main TITAN flange supports the antenna. An additional support has been installed on the tip of the antenna to decrease the static mechanical deformation. The geometry chosen for the support of the antenna and the routing of the coaxial feeding lines were mainly dictated by the clearance needed for the installation in TITAN. It has been decided to install the antenna without using dedicated tools inside the vessel. A lifting structure with a counterweight has been attached to the flange and it is used for displacing the antenna and for the installation [22]. Figure 2 shows also the main features of the mock-up: The 6 straps, the feeding coaxial lines, the trimmers and the diagnostic probes. The feeding lines join the first and last straps using tapered connections (taps). A circuit representation of the installation is presented in figure 3a. More details on the RF circuit and its simulation could be found in [24].

Figure $3 b, c$ show a schematic view of the circuit used for the test and the respective CAD view of the mock-up test setup in the TITAN area. The TWA mock-up is installed inside the TITAN vessel. Two WEST ICRH coaxial vacuum feedthroughs (see Fig. 3b) are used to connect the input and output lines. The transmission lines of the installation are standard $30 \mathrm{ohm}, 9$ inches coaxial lines from Spinner. The input line is connected to a $2 \mathrm{MW}$ RF power source from Thales Electron Devices. The output line is connected to a water load rated for $2 \mathrm{MW}$ operations (also from Thales Electron Devices). Two bi-directional couplers (9 inches, $30 \mathrm{ohm}$, from Spinner) are inserted before the vacuum feedthroughs to provide measurements of the input and output power (see Fig. 3b).

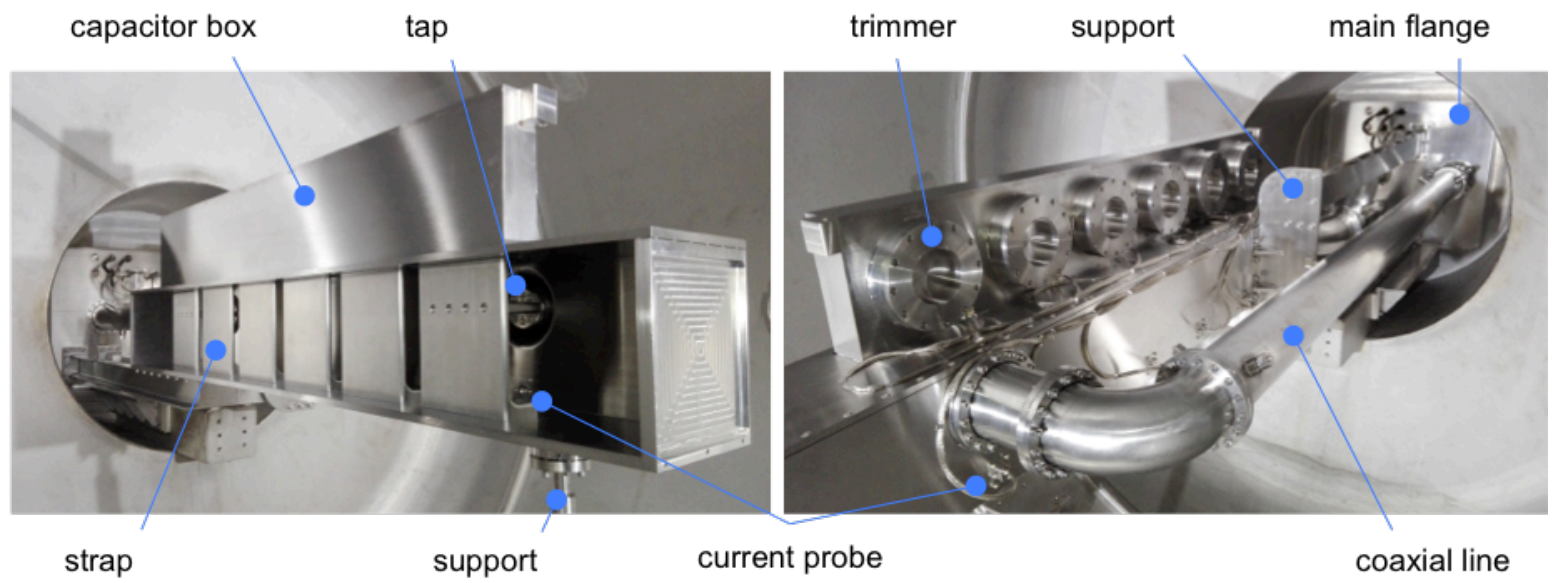

FIG. 2 (left) Front and (right) rear view of the TWA mock-up installed in TITAN. The main flange supports the antenna and an additional vertical support is installed to reduce the deformation. Current and voltage probes are installed for each strap and connected to the main flange. Two $30 \mathrm{ohm}$ coaxial lines are connected to the first and last strap using tapered connections (taps). Each strap is equipped with a trimmer for fine-tuning. 


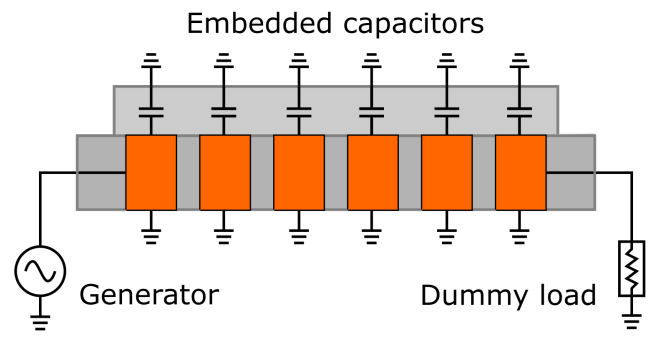

(a)

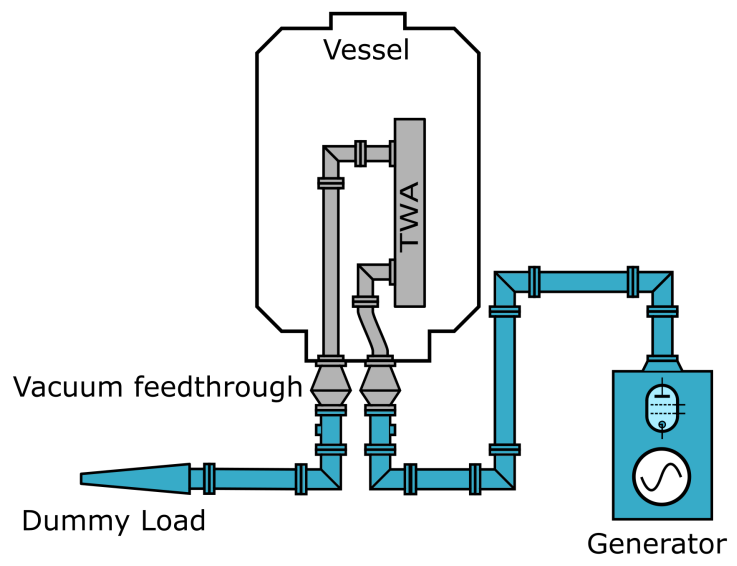

(b)

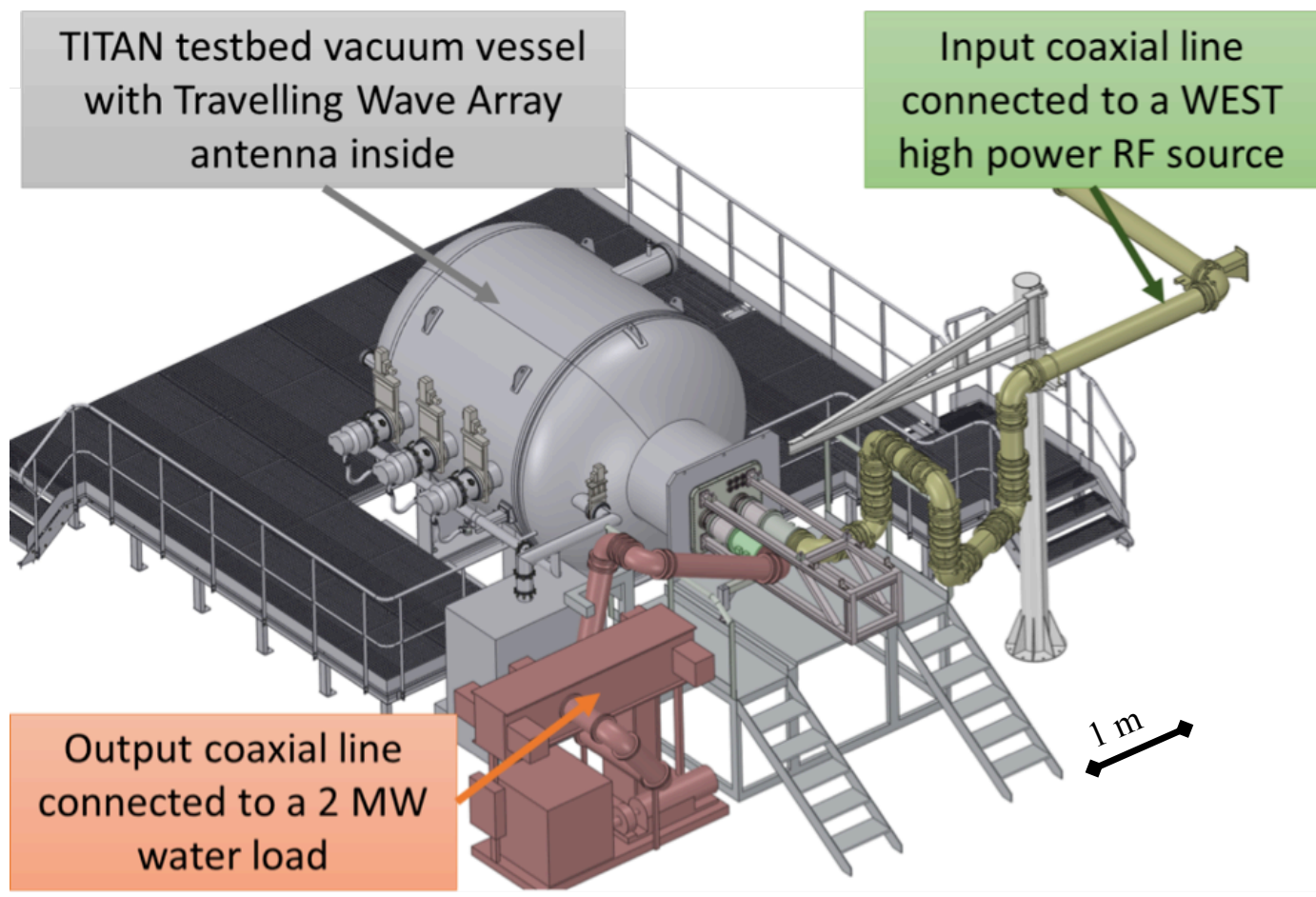

(c)

FIG. 3 (a) RF circuit schematic of the mock-up. The first and last straps are connected to the generator and the dummy load respectively. Each strap has its own embedded capacitor. (b) Schematic representation of the realized circuit. Behind the vacuum feedthroughs, two bi-directional couplers provides a measurement of the input/output power flow. (c) CAD view of the TWA setup for the test in TITAN. The TWA mock-up is installed inside the TITAN vessel. The input coaxial line is connected to one of the $2 \mathrm{MW} R \mathrm{R}$ sources of the WEST installation. The output coaxial line is connected to the $2 \mathrm{MW}$ water load.

\section{MOCK-UP LOW POWER MEASUREMENT}

The mock-up has been tested with a vector network analyser (VNA) outside the TITAN vessel. Initial measurements agreed well with modelling, with minor differences originating from the non-perfect match between the real object and the model used in the simulation. Figure 4a shows the comparison between the measured response and a simulation with similar boundary conditions. The model was above a PEC ground plane with open boundaries (radiation). While the model reproduces quite well the bandwidth of the mock-up, it shows a 
discrepancy in the lower frequency part. The measurement is named out-of-the-box because it was done just after unpacking the structure from its shipping box. Unfortunately, two vacuum leaks were found during the installation of the mock-up in TITAN and the antenna had to be dismounted. One leak has been detected on the main flange and was due to an error in the machining of the vacuum feedthrough connection groove. Proper machining of the groove solved the issue. The second leak occurred in the inner coaxial line, dummy load side, in the elbow before the connection with the tap. It was due to an error in the manufacturing drawings resulting in through instead of passing mounting holes. Welding of threaded rods solved the issue. After reassembling the mock-up, low power measurements with the VNA have been carried out to re-assess the RF response and to investigate the sensitivity to mechanical deformations before and after baking. Figure $4 \mathrm{~b}$ shows the comparison between measurement and simulation of the mock-up, once installed inside the TITAN vessel. They agree well with minor differences with negligible impact on the performance.

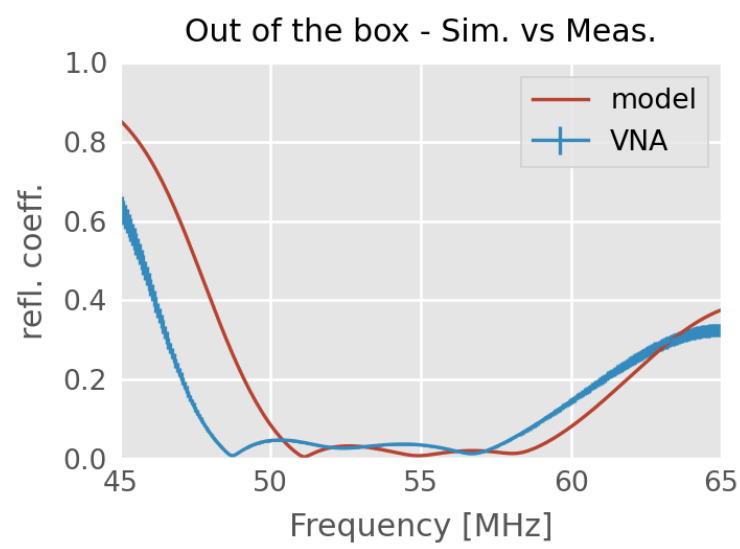

(a)

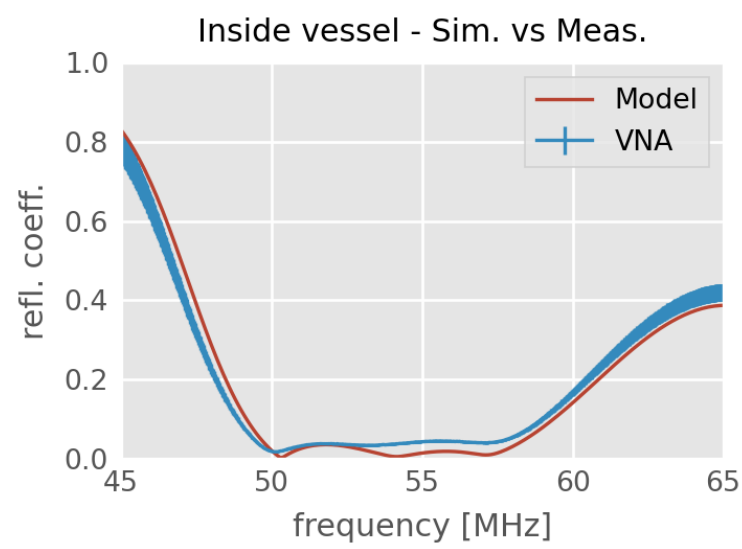

(b)

FIG. 4 Comparison between simulation and measurement of the mock-up response. (a) out-of-the-box measurement. The model captures the bandwith response but shows a shift towards lower frequencies. Measurement and simulations are for the mock-up outside the vessel. (b) For the inside vessel case simulation and measurement agree well.

Trimmers at each strap have been added to allow fine-tuning of the response, mainly for compensating deformations or differences resulting from manufacturing the components. We have adopted two types of trimmers: one made out of brass, movable with a fine thread of $2 \mathrm{~mm}$ per revolution and one made out of stainless steel where the setting is adjusted by machining the surface. The first type is used to easily trim the antenna during the low power measurements. The second type is used for the high power tests. The trimmers allow varying the capacitance of each strap by a few picofarads, which is sufficient to optimally tune the full antenna array. An example of the impact of the trimmers on the response of the antenna is shown in figure 5. The RF response with and without the 6 trimmers is compared. Removing the trimmers, the reactance on each strap is decreased resulting in a shift of the bandwidth towards higher frequencies. The attentive reader will notice that the response is not simply shifted; it is also slightly modified. This is due to the straps not having the same capacitor length [24], as shown in figure 6. Removing the trimmers does not conserve the ratio between the 3 sets of capacitors resulting in a modification of the response. However, the effect is small and appreciable only when the reflection coefficient is expressed in $\mathrm{dB}$. Figure 7 shows the response after fine-tuning the antenna by first using adjustable trimmers and adjusting them and then replacing them with fixed capacitors with the correct value determined in the first step. These measurements were taken outside the TITAN vessel, 
before installation. The final value of the capacitors is a compromise between a good antenna response and the smallest deviation from the optimal value determined with the adjustable trimmers. Here, only three trimmers were modified with changes between $\sim 0.5$ and $\sim 3 \mathrm{~mm}$.

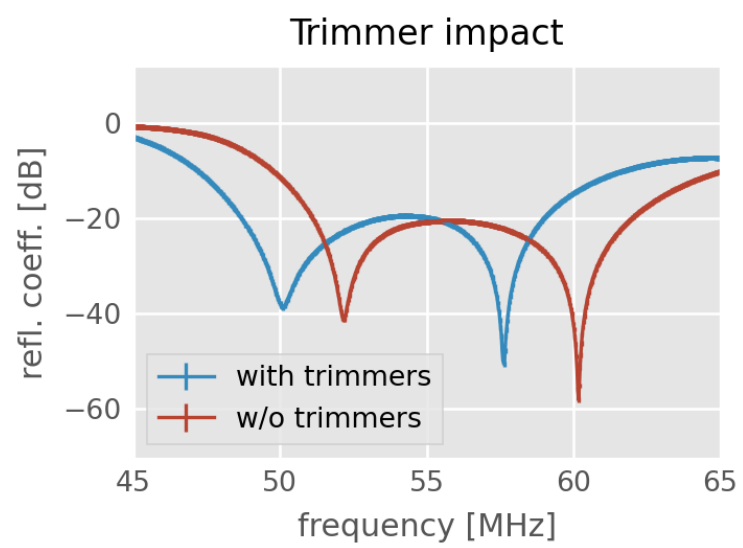

FIG. 5 Measured reflection coefficient of the mock-up. Removing the trimmers decreases the capacitance on each resonator/strap, shifting the band towards higher frequencies.

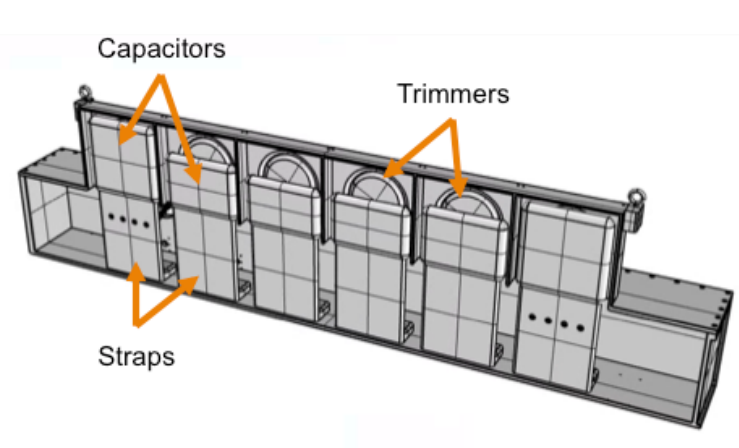

FIG. 6 Cut-plane view of the mock-up CAD model showing the different lengths of the capacitors. The system is symmetric w.r.t. the central axis and has three types (lengths) of strap/capacitor. The trimmers coverage ratio is different for each type.

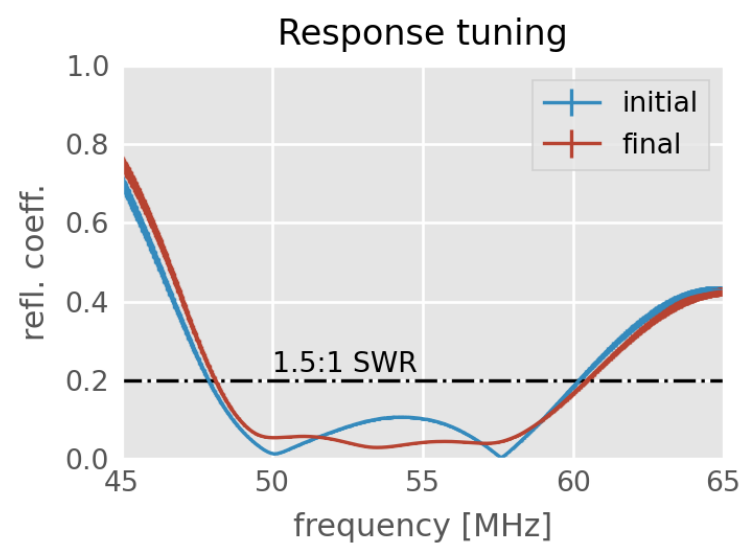

FIG. 7 Fine-tuning of the mock-up response using the trimmers. The final configuration is a compromise between a good response and the smallest difference in the trimmers lengths. These measurements were taken with the mock-up outside the TITAN vessel.

The sensitivity of the antenna to deformations similar to the one that might occur in a reactor environment has been assessed numerically [24]. An initial experimental investigation has been carried out in TITAN, by monitoring the evolution of the RF response as a function of the temperature of the antenna during baking operation. The TITAN vessel was heated up to $240{ }^{\circ} \mathrm{C}$ for about 24 hours. The mock-up was not directly heated by heating elements. It was heated up by radiation from the vessel. The temperature was monitored by 3 thermocouples installed: i) on the outer wall of the input coaxial line (inside the vessel) ii) on the first strap and iii) on the fourth strap. An IR camera looking on the mock-up from a small 
lateral window monitored this last strap. After approximately $12 \mathrm{~h}$ of baking, all the monitored components of the antenna reached a temperature above $200{ }^{\circ} \mathrm{C}$. Figure 8 shows the negligible difference between the RF response at $25{ }^{\circ} \mathrm{C}$ and $200{ }^{\circ} \mathrm{C}$ (measured at the strap). This result shows the resilience of the antenna to thermal deformation similar to the one that will occur in a reactor blanket when heated up from room temperature to the operating temperature, which is in the order of several hundreds of ${ }^{\circ} \mathrm{C}$. Comparing figure 7 with figure 8 , there is small differences between the final configuration trace and the trace measured at room temperature during the baking phase. The discrepancy is due to the different external conditions. For instance, the measurements reported in figure 7 were taken before installing the mock-up into the vessel. Moreover, the mock-up was lifted for the installation and slight mechanical deformations occurred during this operation. Those deformations were due to the specific support strategy used for the mock-up resulting in a long cantilever beam. However, the differences are small and do not have any significant effect on the performance of the system.

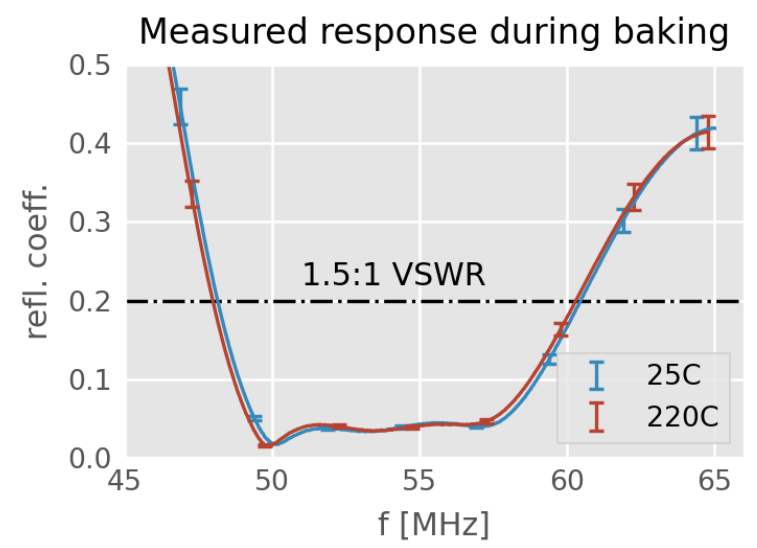

FIG. 8 Measured RF response (reflection coefficient) of the mock-up at room temperature $\left(25^{\circ} \mathrm{C}\right)$ and at baking temperature $\left(>200^{\circ} \mathrm{C}\right)$. The response does not change significantly after the thermo-mechanical deformation. In the figure is also indicated the level of SWR 1.5:1, considered as the limit for the antenna operation, imposed by the generator limits at high power.

\section{MOCK-UP DIAGNOSTICS}

The voltage probes consisted of coaxial vacuum feedthroughs, as shown in figure 9, where the inner pin is the coupling electrode. The insulation was made of PEEK and the probe was rated for operation at $250{ }^{\circ} \mathrm{C}$. A $2 \mathrm{D}$ electrostatic model of the probe has been used to model its performance. The calibration procedure required isolation of the straps from the antenna box, achieved using a thin sheet of Kapton. The strap was then excited at a frequency between $300 \mathrm{kHz}<\mathrm{f}<2 \mathrm{MHz}$ and the response of the voltage probe was recorded. The measured response was then transformed in a frequency dependant attenuation factor, which resulted in a coefficient that links the probe voltage to the voltage on the strap. In a second step, the Kapton isolated coaxial cables that connect the voltage probes to the flange are calibrated. The data acquisition system developed for these experiments made use of two types of integrated circuits: the AD8310 as a logarithmic amplifier for the measurement of the strap voltage amplitude and the AD8302 as a gain/phase detector for the measurement of the strap current amplitude and phase. Current probes consisting of small loops installed on the back of the antenna box have been installed close to the short circuit of the strap. A 
picture of one of those probes and their positions is shown in figure 9. For those current probes we rely on a "synthetic" calibration: the probe itself was modelled in Ansys HFSS simulating an ideal calibration setup. The cables used for the current probes were the same as the cables used for the voltage probes. Measurements of the cables gave the calibration factors for the phase.

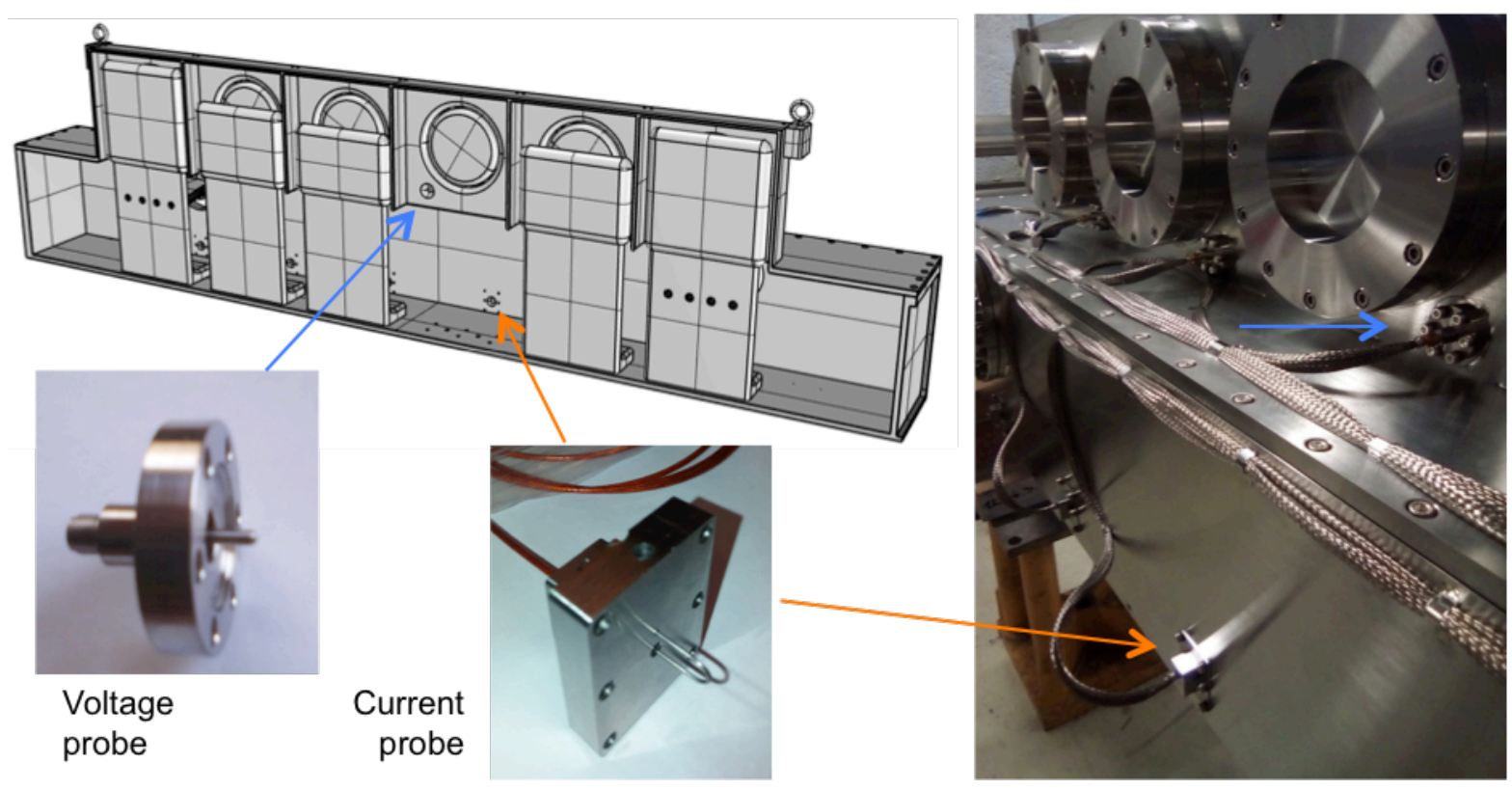

FIG. 9 Cut-plane view of the mock-up CAD model showing the location of the voltage and current probes, installed for each strap. One strap is not displayed to allow locating the probes. On the right, a picture of the back of the mock-up indicates the locations of the probes. The voltage probes are made of vacuum feedthroughs and are positioned in the bottom part of each capacitor box, below the trimmers. The current probes are small loops and are mounted on the back of the antenna box, close to the short circuit of the straps.

Particular care has been taken during the production and installation of the probes. Each loop measures $8 \mathrm{~mm} \times 15 \mathrm{~mm}$ with a $1.3 \mathrm{~mm}$ diameter wire. The simulated attenuation of the probe was $\sim 65 \mathrm{~dB}$.

Low power assessments validated the measurement chain. For the tests, a $55 \mathrm{~dB}$ amplifier was connected to the vector network analyser (VNA). The output was then connected to a 50 to $30 \mathrm{ohm}$ matching unit made of two stub-line sections. This setup allows injecting up to $50 \mathrm{~W}$ of power, mainly limited by the available cables (RG58). However, the main limitation of the setup was the $30 \mathrm{ohm}$ load connected to the output line, rated for $1 \mathrm{~W}$. The output power of the VNA alone was not sufficient for the dynamic range of the acquisition card. We thus set a power of $30 \mathrm{dBm}(1 \mathrm{~W})$, monitored by a directional coupler inline with the output of the amplifier connected to a power meter.

\section{MOCK-UP PRELIMINARY HIGH POWER RESULTS}

High power tests of the mock-up were carried out after the baking phase by first injecting power in the range of $50-100 \mathrm{~kW}$, for short time $(\sim 10-50 \mathrm{~ms})$ while monitoring the outgassing of the structure. No substantial outgassing was registered. To characterize the response of the system, a set of short pulses was performed at a power level of $200 \mathrm{~kW}$, scanning the whole available bands of the generator, covering from $47 \mathrm{MHz}$ up to $65 \mathrm{MHz}$, with a gap at $59 \mathrm{MHz}<\mathrm{f}<61 \mathrm{MHz}$. This power level allows measuring the response of the 
antenna across the whole generator band, which is larger than the antenna bandwidth while keeping a good signal-to-noise ratio. Outside of the antenna bandwidth, the reflection coefficient is too large for high power operation, i.e. the standing wave ratio SWR $>1.5: 1$. However, with an injected power of $200 \mathrm{~kW}$, the reflected power is always below the maximum tolerable by the generator. The forward and reflected powers were measured just before the vacuum feedthrough, employing a directional coupler (see Fig. 3b), and used to calculate the reflection coefficient of the antenna. Figure 10 shows the comparison between the reference scan, measured at room temperature, and a scan performed after a stress test consisting of 5 pulses of $30 \mathrm{~s}$ duration, $25 \%$ duty cycle, with an injected power of $500 \mathrm{~kW}$. This set of pulses was used to increase the temperature of the straps and the feeding lines by ohmic losses, exploiting the fact that the mock-up is not water-cooled. The localized temperature increase induced some thermo-mechanical deformations. A subsequent scan of the band did not reveal a substantial difference with the reference scan. Further shots to enhance the deformation were not considered necessary. Figure 8 and 10 combined show that the mock-up is resilient to thermal deformations and that the antenna response is under control.

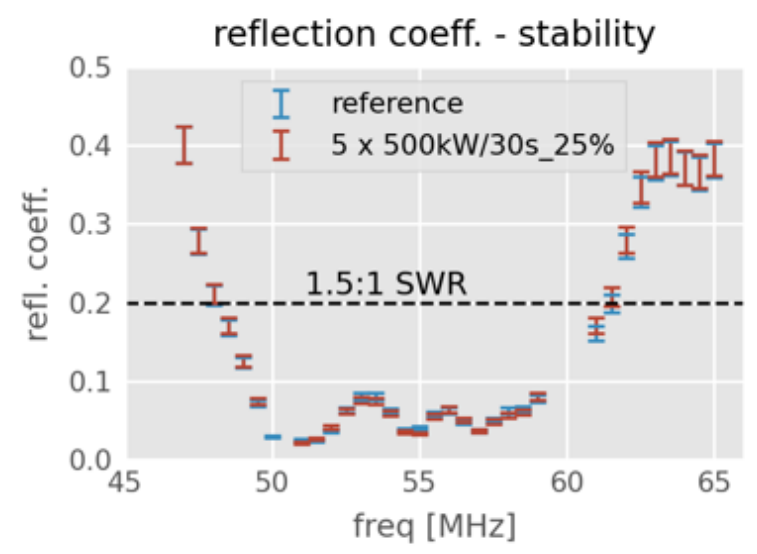

FIG. 10 Measurement of the reflection coefficient for two frequency scans in the generator bands, interleaved with a set of 5 pulses at $500 \mathrm{~kW} / 30 \mathrm{~s}$ at $25 \%$ duty cycle used to induce thermo-mechanical deformation in the antenna. The injected power was $200 \mathrm{~kW}$ for a few ms in both cases. This power level allows to measure the response also in the region where high power operation of the generator is not allowed, i.e. for an SWR $>1.5: 1$ (dashed line).

Subsequently, the power was raised in steps up to $2 \mathrm{MW}$ and several shots were performed for different time durations at $55.5 \mathrm{MHz}$. No sign of tripping of the generator due to arcs in the antenna was observed in any shot. $55.5 \mathrm{MHz}$ is the standard operating frequency of the WEST antennas and the generator is optimised and well tested at this frequency. We decided to use this value for operations above $500 \mathrm{~kW}$ to avoid unnecessary risks for the power plant. Figure 11 presents the case for $2 \mathrm{MW} / 1 \mathrm{~s}$ flattop. The figure shows the time evolution of the generator forward and reflected power. From figure 10 the reflection coefficient is $\sim 0.06$ at $55.5 \mathrm{MHz}$ and the measured reflected power is $\sim 7 \mathrm{~kW}$. The net power flowing at the input of the antenna is thus practically the same as the generator forward power The slope of the power waveform was limited to $\sim 500 \mathrm{~kW} / \mathrm{s}$ by the generator control system. The power losses are estimated by comparing the measurements of the mock-up input and output power, considering negligible the contribution of radiated power. Figure 12 shows the fraction of power lost in the antenna, calculated as $1-\left(\mathrm{P}_{\text {out }} / \mathrm{P}_{\text {in }}\right)$ where $\mathrm{P}_{\text {in }}$ is the net input power, i.e. $\mathrm{P}_{\mathrm{fwd}}-\mathrm{P}_{\mathrm{rfl}}$. The power loss fraction is below $4 \%$. It has to be noted that the antenna is made of bare stainless steel without additional coating. 


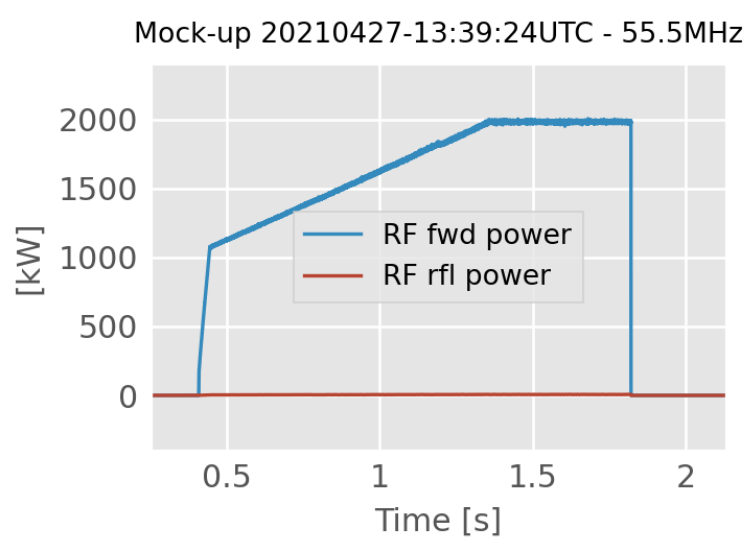

FIG. 11 Measured RF forward power flowing in the TWA mock-up for a $2 \mathrm{MW} / 1$ s flattop test. The reflected power is $\sim 7 \mathrm{~kW}$ resulting in a net power flow in the antenna equal to the generator forward power.

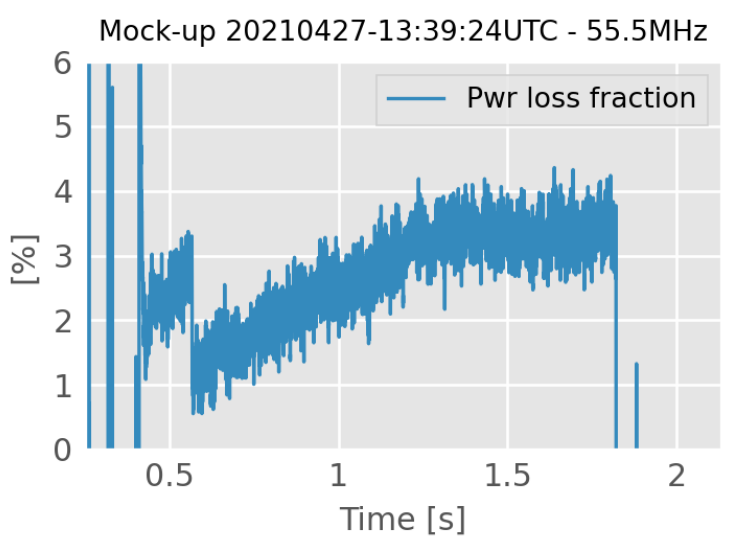

FIG. 12 Estimation of the power lost in the TWA mock-up for a $2 \mathrm{MW} / 1$ s flattop test, calculated as $1-\left(P_{\text {out }} / P_{\text {in }}\right)$.

The other tests performed that qualify the mock-up for WEST scenarios are: $500 \mathrm{~kW} /$ $60 \mathrm{~s}, 1 \mathrm{MW} / 20 \mathrm{~s}$ and $1.75 \mathrm{MW} / 5 \mathrm{~s}$. Figure 13 shows the time traces of the representative parameters monitored during one of the $500 \mathrm{~kW} / 60 \mathrm{~s}$ tests. Those are the forward power, the voltages on the straps, the reflection coefficient (expressed in $\mathrm{dB}$ as return loss) and the pressure in the vessel. The reflected power is well below the generator limit and can be easily calculated from the return loss trace. The return loss is defined as $10 * \log _{10}\left(\mathrm{P}_{\text {out }} / \mathrm{P}_{\text {in }}\right)$. Its mean value is $\sim-22 \mathrm{~dB}$ which corresponds to $\sim 0.6 \%$ of reflected power. Return loss tends to decrease during the pulse, probably due to thermo-mechanical deformations. The pressure does increase slightly due to the increase in temperature due to ohmic losses. As a reminder, the mock-up is not actively cooled. 


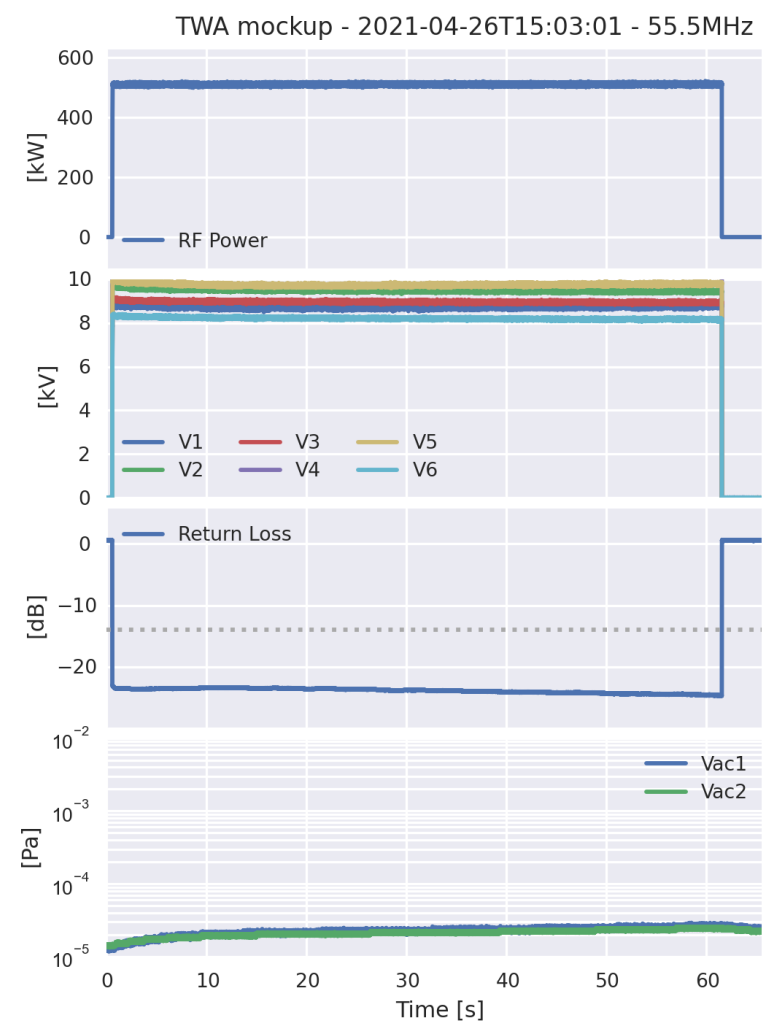

FIG. 13 Measured parameters during one of the $500 \mathrm{~kW} / 60 \mathrm{~s}$ tests. From the top, time traces of: RF forward power, voltage on the straps, reflection coefficient and chamber pressure. The reflected power, monitored through the reflection coefficient, is well below the generator limit, displayed as a dotted line, and shows a tendency to further decrease during the pulse. The pressure increased slightly due to the increase in the temperature of the structure.

\section{EXTRAPOLATION TO DEMO}

The aim of the test of a TWA on WEST is to assess the performance of this antenna type under reactor relevant conditions. In this respect, WEST is a rather unique machine with its long pulse $(1000 \mathrm{~s})$ operation capabilities and full metallic walls. The WEST TWA has been designed such that it would allow a first careful extrapolation to the reactor. Figure 14 shows a possible implementation of a distributed ICRH antenna system based on the TWA concept. Each machine sector (16, for DEMO 2019) is made of 3 outboard blanket modules: one central and two lateral ones. The TWA structures are placed in the lateral modules, poloidally displaced to not interfere with the other systems, as shown in figure 14 by the neutral beam injector (NBI) duct and the equatorial limiter. 

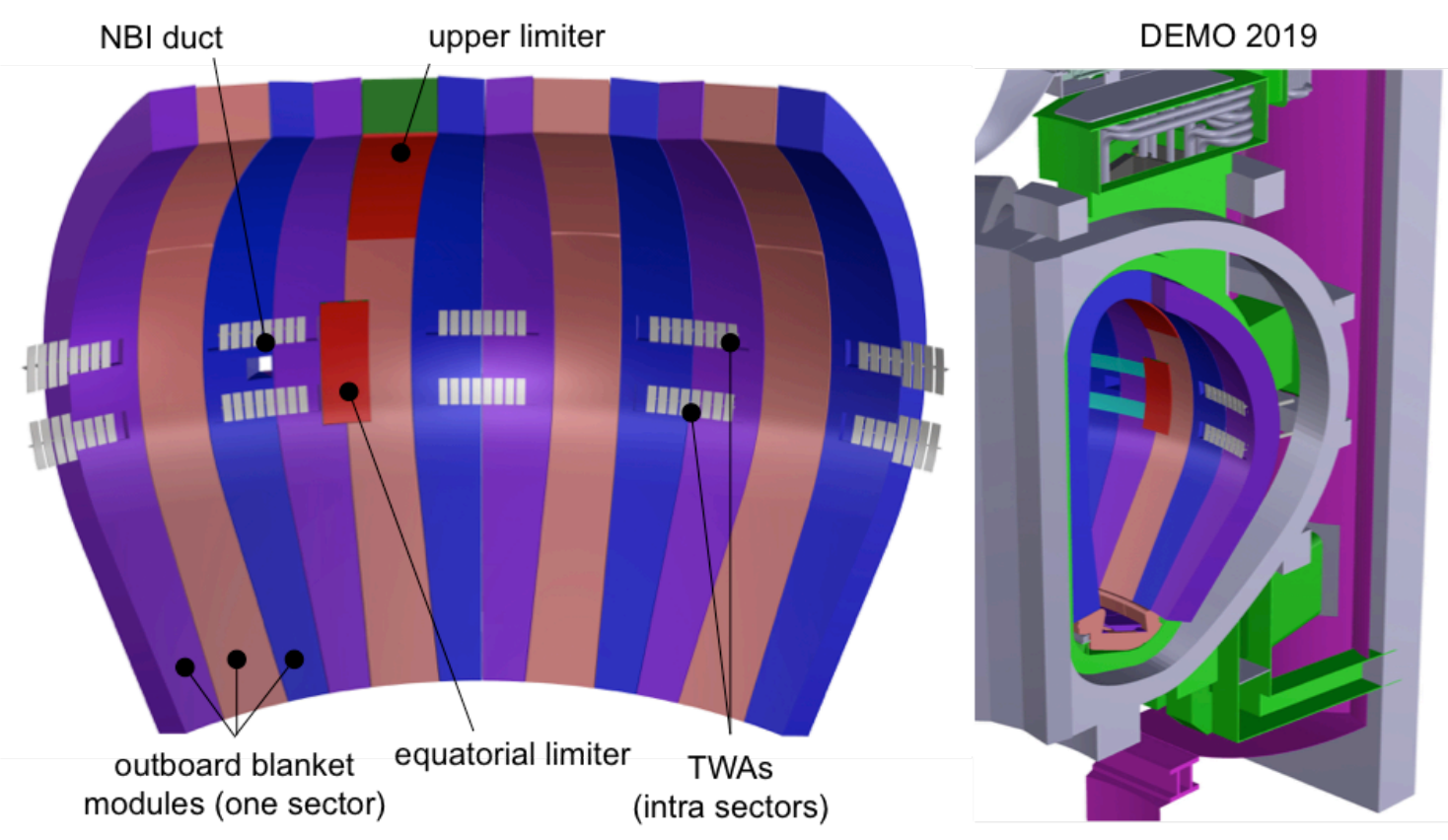

FIG. 14 (right) 3D view of the DEMO 2019 CAD model showing a 36 deg sector of the machine. (left) CAD model of 4 outboard blanket sectors, each composed of 3 modules. The TWAs are integrated into the lateral modules of each sector (blue and violet) and displaced poloidally to not interfere with the other systems in the equatorial plane (NBI and limiter shown in the figure).

Each half of an antenna is integrated into one blanket module. This means that the antenna straps play effectively the role of first wall components and thus their design has to follow the same criteria. Moreover, the antenna shares the cooling of the blanket modules. The only penetrations required are the feeding coaxial lines, two for each module. Considering a nominal power of $50 \mathrm{MW}$, and a target profile like ITER-2010-low [13,14], each antenna could be rated at $\sim 2-2.5 \mathrm{MW}$ of input power. The mock-up validated in TITAN and the antennas foreseen in WEST are designed for the same power performance. Another important aspect is the sensitivity of the antenna to mechanical and thermal deformations. The tested mock-up, which is not water-cooled, has shown that the RF response of the antenna was little depending on mechanical and thermal deformations. In a future step, the foreseen TWA antenna on WEST will complement those results by adding the effect of plasma radiation and particle fluxes.

\section{DISCUSSION AND CONCLUSIONS}

The WEST TWA antenna design has been updated to limit the port usage to a single port. Although the main reason behind this development is the reinstallation of an ECRH system on WEST, this is nevertheless a good opportunity to tackle some of the risks identified from the previous studies, in particular those related to the current loop across two machine sectors, using two vessel ports to feed the $2.5 \mathrm{~m}$ long antenna. Using only one port also opens up the possibility to implement a radial movement of the antenna. The two TWA rows are displaced poloidally, which simplifies the routing of the feeding lines and allows installing additional diagnostics. The reduced antenna length and the number of straps for the updated antenna design will lead to changes in the results obtained so far and require additional studies. While the power load will decrease due to the decrease of the antenna 
surface, the amount of RF power will increase to compensate for the loss of one or two straps. No changes are expected on the external components. Further integration studies are required to assess the clearance with the ECRH beam and its potential impact on the plasma SOL.

The TWA mock-up has been installed in TITAN and its diagnostic system was commissioned. To monitor the thermal evolution of the antenna, 3 thermocouples are installed; one on the support structure and two inside two of the straps. The effect of mechanical and thermal deformations on the antenna response has been studied and validate the modellings. The use of trimmers to fine-tune the antenna response was useful to fine-tune the antenna RF response after the subsequent assembling/reassembling required to fix the vacuum leaks. The installed antenna mock-up has a response that agrees well with the modelling results and the requirements for the validation tests.

The antenna was baked to $200^{\circ} \mathrm{C}$ while monitoring its $\mathrm{RF}$ response. The results provided information on the sensitivity to thermal deformations that can be extrapolated for the reactor case. RF conditioning was performed without noticing any sign of arcs and tripping of the generators. The power was increased up to the maximum level available at the generator: $2 \mathrm{MW}$. In particular, successful shots at $500 \mathrm{~kW} / 60 \mathrm{~s}, 1 \mathrm{MW} / 20 \mathrm{~s}, 1.75 \mathrm{MW} / 5$ $\mathrm{s}$ and $2 \mathrm{MW} / 3 \mathrm{~s}$ confirm the property of the mock-up and open the way for a WEST TWA antenna. The RF response was measured at several frequencies across and outside the frequency band at $\mathrm{P}<200 \mathrm{~kW}$, which is the maximum reflected power accepted by the generator. The measured response of the antenna in the bandwidth of the RF generator (i.e. 47-65 MHz) qualified the mock-up and the design strategy.

\section{ACKNOWLEDGEMENTS}

This work has been carried out within the framework of the EUROfusion Consortium and has received funding from the Euratom research and training programme 2014-2018 and 2019-2020 under grant agreement No 633053. The views and opinions expressed herein do not necessarily reflect those of the European Commission.

Part of this work was carried out under the framework of the Sino-French Fusion Energy centeR (SIFFER) http://www.siffer.science/.

This research made use of scikit-rf, an open-source Python package for RF and Microwave applications.

\section{REFERENCES}

[1] VAN EESTER, D., et al., Ion cyclotron resonance heating scenarios for DEMO, Nucl. Fusion 59 (2019) 106051

[2] DOUAI D., et al., Wall conditioning for ITER: Current experimental and modeling activities, Journal of Nuclear Materials, 463 (2015) 150-156

[3] JOFFRIN E., et al., Overview of the JET preparation for deuterium-tritium operation with the ITER like-wall, Nucl. Fusion 59 (2019) 112021

[4] FIELD A. R., et al., The impact of fuelling and W radiation on the performance of high-power, ITER-baseline scenario plasmas in JET-ILW, Plasma Phys. Control. Fusion 63 (2021) 095013

[5] LERCHE E. et al., Sawtooth control with modulated ICRH in JET-ILW H-mode plasmas Nucl. Fusion 60 (2020) 126037

[6] HOBIRK J., et al., Analysis of plasma termination in the JET hybrid scenario, Nucl. Fusion 58 (2018) 076027

[7] SAUTER O., et al., ITER baseline scenario investigations on TCV and comparison with AUG, 28th IAEA Fusion Energy Conference (2021)

[8] SOZZI C., et al., Termination of discharges in high performance scenarios in JET, 28th IAEA Fusion Energy Conference (2021) 
[9] FRANKE, T., et al., Review of the Innovative H\&CD Designs and the Impact of Their Configurations on the Performance of the EU DEMO Fusion Power Plant Reactor, IEEE Transaction on Plasma Science 46 (2018) 1633-1640

[10] NOTERDAEME, J.M., et al., Progress on an ion cyclotron range of frequency system for DEMO, Fusion Eng. Des. 146 A (2019) 1321-1324

[11] MESSIAEN A. and MAQUET V., Coaxial and surface mode excitation by an ICRF antenna in large machines like DEMO and ITER, Nucl. Fusion 60 (2020) 076014

[12] MAQUET V. and MESSIAEN A., Optimized phasing conditions to avoid edge mode excitation by ICRH antennas. Journal of Plasma Physics, 86(6) (2020) 85586060

[13] RAGONA, R., et al., A travelling wave array system as solution for the ion cyclotron resonance frequencies heating of DEMO, Nucl. Fusion 60 (2020) 016027

[14] MESSIAEN, A., RAGONA, R., Study of the coupling of distributed ICRH antenna and of its optimisation for heating of large machines as DEMO, 43rd EPS Conf. on Plasma Physics, 4-8 July 2016, Leuven BE

[15] MOELLER, C.P., et al., Combline antennas for launching traveling fast waves, AIP Conference Proceedings 289 (1994) 323

[16] OGAWA, T., et al., Radiofrequency experiments in JFT-2M: Demonstration of innovative applications of a travelling wave antenna, Nucl. Fusion 41 (2001) 1767

[17] WI, H.H., et al., Design and RF test of a prototype traveling wave antenna for helicon current drive in KSTAR, Fusion Eng. Des. 126 (2018) 67-72

[18] TOOKER, J.F., et al., Development of a high power Helicon system for DIII-D, Fusion Eng. Des. 123 (2017) 228-231

[19] JO, J-G., et al., Coupling study of fast wave near the lower hybrid frequency range in VEST, Physics of Plasmas 25 (2018) 082511

[20] RAGONA, R., et al., Traveling wave array for DEMO with proof of principle on WEST, Fusion Eng. Des. 146 A (2019) 854-85

[21] RAGONA, R., et al., Progress on the design of a DEMO high power ICRH travelling wave antenna mock-up to be tested on WEST, AIP Conference Proceedings 2254 (2020) 070014

[22] BATAL, T., et al., Design and Thermal-structural analysis of a high power ICRH Travelling Wave Array antennas, Fusion Eng. Des. 166 (2021) 112325

[23] BERNARD, J-M., et al., New high power CW test facilities for ITER ICRH components testing, Fusion Eng. Des. 86 (2011) 876-879

[24] RAGONA, R., et al., RF network analysis of the WEST TWA mock-up in TITAN, Fusion Eng. Des. 168 (2021) 112615 